\title{
DEFICIENT VALUES AND ANGULAR DISTRIBUTION OF ENTIRE FUNCTIONS
}

\author{
LO YANG
}

\begin{abstract}
Let $f(z)$ be an entire function of positive and finite order $\mu$. If $f(z)$ has a finite number of Borel directions of order $\geq \mu$, then the sum of numbers of finite nonzero deficient values of $f(z)$ and all its primitives does not exceed $2 \mu$. The proof is based on several lemmas and application of harmonic measure.
\end{abstract}

1. Introduction. Let $f(z)$ be an arbitrary entire function of finite and positive order $\lambda$. A. Denjoy conjectured that $f(z)$ has at most $2 \lambda$ distinct asymptotic values. L. Ahlfors succeeded in proving this conjecture later. He proved further that any entire function of finite lower order $\mu$ has $2 \mu$ asymptotic values at most [6, pp. 303-307].

Since there are some similarities between asymptotic values and deficient values of entire functions, one may expect that any entire function of finite order would have a finite number of deficient values. N. U. Arakelyan [1], however, constructed for the first time an entire function of order one having infinite deficient values.

It is natural to ask: for an entire or meromorphic function of finite order $\lambda$ or finite lower order $\mu$, under what conditions does the number of its deficient values have an upper bound depending only on $\lambda$ or $\mu$ ? We ask for conditions which ensure that the number of deficient values of an entire function satisfy the bound of Ahlfors's theorem.

One such condition is that entire or meromorphic functions have deficiency sum two. This is actually a part of F. Nevanlinna's conjecture. In the case of entire functions, cf. A. Pfluger [7], A. Edrei and W. Fuchs [4] proved several interesting theorems. Then A. Weitsman [10] obtained partial results for meromorphic functions and D. Drasin $[\mathbf{2}, \mathbf{3}]$ recently made a great contribution to prove F. Nevanlinna's conjecture completely. For entire functions, we also posed a similar condition [13]

$$
\sum_{j=-\infty}^{+\infty} \sum_{a \neq 0, \infty} \delta\left(a, f^{(j)}\right)=1
$$

and obtained the corresponding result.

In this paper, another condition will be introduced.

Let $f(z)$ be an entire function of lower order $\mu$, where $0<\mu<\infty$. A ray $\arg z=\theta_{0}\left(0 \leq \theta_{0}<2 \pi\right)$ is named a Borel direction of order $\geq \mu$ of $f(z)$, if for any

Received by the editors January 15,1987 . The contents of this paper were presented on July 8, 1987, to Research Symposium on Complex Analysis to celebrate Professor W. K. Hayman's sixtieth birthday. The abstract was announced in Kexue Tongbao (Science Bulletin) 33 (1988).

1980 Mathematics Subject Classification (1985 Revision). Primary 30D35. 
positive number $\varepsilon$, the inequality

$$
\varlimsup_{r \rightarrow+\infty} \frac{\log n\left(r, \theta_{0}-\varepsilon, \theta_{0}+\varepsilon, f=a\right)}{\log r} \geq \mu
$$

holds for any finite complex value $a$, with possibly one exceptional value, where $n\left(r, \theta_{0}-\varepsilon, \theta_{0}+\varepsilon, f=a\right)$ denotes the number of zeros of $f(z)-a$ in the region $(|z| \leq r) \cap\left(\theta_{0}-\varepsilon \leq \arg z \leq \theta_{0}+\varepsilon\right)$, multiple zeros being counted with their multiplicities.

The aim of this paper is to prove the following theorem.

THEOREM. Let $f(z)$ be an entire function of lower order $\mu$, where $0<\mu<$ $+\infty$. If $q<\infty$ is the number of Borel directions of order $\geq \mu$ of $f(z)$ and $p_{l}$ $(l=0,-1,-2, \ldots)$ denotes the number of finite nonzero deficient values of $f^{(l)}(z)$ $\left(l=0,-1,-2, \ldots ; f^{(0)} \equiv f(z)\right),{ }^{1}$ then we have $\sum_{l=0}^{-\infty} p_{l} \leq 2 \mu$.

\section{A property of monotonic functions.}

2.1. We first establish a lemma on monotonic functions.

LEMMA 1. Let $f(z)$ be an entire function of finite lower order $\mu$, where $0<$ $\mu<+\infty$. If $\beta_{1}$ and $\beta_{2}$ are two arbitrary numbers with $1<\beta_{1}<\beta_{2}$, then there exists a sequence $\left\{t_{k}\right\}$ of positive numbers tending to infinity such that

$$
\lim _{k \rightarrow+\infty} \frac{\log T\left(t_{k}, f\right)}{\log t_{k}}=\mu
$$

and the inequalities

$$
T\left(\beta_{1} t_{k}, f\right)<\left(\beta_{1}^{\mu}+o(1)\right) T\left(t_{k}, f\right)
$$

and

$$
T\left(\beta_{2} t_{k}, f\right)<\left(\beta_{1}^{\mu} \beta_{2}^{\mu}+o(1)\right) T\left(t_{k}, f\right)
$$

hold, when $k$ tends to infinity.

PROOF. Since the lower order of $f(z)$ equals $\mu$, there is a sequence $\left\{r_{k}\right\}(k=$ $2,3, \ldots)$ of positive numbers tending to infinity such that

$$
\left(r_{k}^{1-1 / k}\right)^{\mu-1 / k^{2}}<T\left(r_{k}^{1-1 / k}, f\right) \leq T\left(r_{k}, f\right)<r_{k}^{\mu+1 / k^{2}}
$$

and

$$
\log r_{k}>k^{3}(\mu+2 / k) \log \left(\beta_{1} \beta_{2}\right) .
$$

We shall prove the existence of a corresponding sequence $\left\{t_{k}\right\}$ which satisfies

$$
\begin{gathered}
t_{k} \in\left[r_{k}^{1-1 / k}, r_{k}\right], \quad k=1,2,3, \ldots, \\
T\left(\beta_{1} t_{k}, f\right)<\beta_{1}^{\mu+2 / k} T\left(t_{k}, f\right)
\end{gathered}
$$

${ }^{1} f^{(l)}(z)(l<0)$ is the primitive of order $|l|$ of $f(z)$. 
and

$$
T\left(\beta_{2} t_{k}, f\right)<\left(\beta_{1} \beta_{2}\right)^{\mu+2 / k} T\left(t_{k}, f\right),
$$

hence the conclusion of lemma.

Let us now prove the existence of $\left\{t_{k}\right\}$ satisfying (2.6), (2.7) and (2.8). Denote

$$
\begin{aligned}
& E=\left\{t: r_{k}^{1-1 / k} \leq t \leq r_{k}, T\left(\beta_{2} t, f\right) \geq\left(\beta_{1} \beta_{2}\right)^{\mu+2 / k} T(t, f)\right\}, \\
& \tau_{1}=\min E, \quad \tau_{j}=\min \left\{E \cap\left[\beta_{2} \tau_{j-1}, r_{k}\right]\right\}, \quad j=2,3, \ldots, J+1,
\end{aligned}
$$

where $J$ is chosen so that $\tau_{J+1}<r_{k} \leq \beta_{2} \tau_{J+1}$. For every $j(0 \leq j \leq J)$ set further

$$
\begin{aligned}
& e_{j}=\left\{t: \beta_{1} \tau_{j} \leq t \leq \tau_{j+1}, T\left(\beta_{1} t, f\right) \geq \beta_{1}^{\mu+2 / k} T(t, f)\right\}, \\
& \sigma_{j, 1}=\min e_{j}, \\
& \sigma_{j, l}=\min \left\{e_{j} \cap\left[\beta_{1} \sigma_{j, l-1}, \tau_{j+1}\right]\right\}, \quad l=2,3, \ldots, L_{j}+1,
\end{aligned}
$$

where $\tau_{0}=r_{k}^{1-1 / k}$ and $\sigma_{j, L_{j}+1}<\tau_{j+1} \leq \beta_{1} \sigma_{j, L_{j}+1}$.

By the above notations and (2.4), we have

$$
\begin{aligned}
\left(r_{k}^{1-1 / k}\right)^{\mu-1 / k^{2}} & <T\left(r_{k}^{1-1 / k}, f\right) \leq T\left(\sigma_{0,1}, f\right) \\
& \leq \beta_{1}^{-(\mu+2 / k)} T\left(\sigma_{0,2}, f\right) \leq \cdots \leq \beta_{1}^{-L_{0}(\mu+2 / k)} T\left(\sigma_{0, L_{0}+1}, f\right) \\
& \leq \beta_{1}^{-L_{0}(\mu+2 / k)} T\left(\tau_{1}, f\right) \leq \beta_{1}^{-L_{0}(\mu+2 / k)}\left(\beta_{1} \beta_{2}\right)^{-(\mu+2 / k)} T\left(\beta_{2} \tau_{1}, f\right) \\
& \leq \cdots \leq \beta_{1}^{-\left(L_{0}+L_{1}\right)(\mu+2 / k)}\left(\beta_{1} \beta_{2}\right)^{-2(\mu+2 / k)} T\left(\beta_{2} \tau_{2}, f\right) \\
& \leq \cdots \leq \beta_{1}^{-(\mu+2 / k) \sum_{j=0}^{J} L_{j}}\left(\beta_{1} \beta_{2}\right)^{-J(\mu+2 / k)} T\left(r_{k}, f\right) \\
& <\beta_{1}^{-(\mu+2 / k) \sum_{j=0}^{J} L_{j}}\left(\beta_{1} \beta_{2}\right)^{-J(\mu+2 / k)} r_{k}^{\mu+1 / k^{2}},
\end{aligned}
$$

so that

$$
\left(\mu+\frac{2}{k}\right) \sum_{j=0}^{J} L_{j} \log \beta_{1}+J\left(\mu+\frac{2}{k}\right) \log \left(\beta_{1} \beta_{2}\right)<\left(\mu+\frac{2}{k}-\frac{1}{k^{2}}\right) \frac{\log r_{k}}{k} .
$$

Thus

$$
\begin{aligned}
& \sum_{j=0}^{J} L_{j} \log \beta_{1}+(J+1) \log \beta_{1}+(J+1) \log \beta_{2} \\
& \quad<\left\{1-\frac{1}{k^{2}\left(\mu+\frac{2}{k}\right)}+\frac{k \log \left(\beta_{1} \beta_{2}\right)}{\log r_{k}}\right\} \frac{\log r_{k}}{k}<\frac{\log r_{k}}{k}
\end{aligned}
$$

by (2.5). Consequently

$$
\int_{E} \frac{d t}{t}+\sum_{j=0}^{J} \int_{e_{j}} \frac{d t}{t}<\int_{r_{k}^{1-1 / k}}^{r_{k}} \frac{d t}{t}
$$

i.e. there is a value $t_{k}$ not in the set $E \cup\left(\bigcup_{j=0}^{J} e_{j}\right)$. 


\section{Several known results.}

3.1. In the sequel, we need several known results.

LEMMA A [12]. Let $f(z)$ be an entire function of lower order $\mu$, where $0<\mu<$ $+\infty$. If $f(z)$ has no Borel direction of order $\geq \mu$ in the angle $\theta_{1}<\arg z<\theta_{2}$, then there exist two finite distinct complex values $a_{j}(j=1,2)$ such that for any number $\varepsilon, 0<\varepsilon<\left(\theta_{2}-\theta_{1}\right) / 2$, we have

$$
\varlimsup_{r \rightarrow+\infty} \frac{\log \left\{\sum_{j=1}^{2} n\left(r, \theta_{1}+\varepsilon, \theta_{2}-\varepsilon, f=a_{j}\right)\right\}}{\log r}<\mu .
$$

LEMMA B [12]. Let $f(z)$ be an entire function of lower order $\mu$, where $0<\mu<$ $+\infty$. If the number of its Borel directions of order $\geq \mu$ is finite and the minimum angular separation of the Borel directions is equal to $\omega$, then the order $\lambda$ of $f(z)$ must be finite and $\mu \leq \lambda \leq \pi / \omega$.

LEMMA C [13, p. 468]. Let $g(\varsigma)$ be meromorphic on $|\varsigma| \leq R(<+\infty)$. If

$$
\mathfrak{N}=n(R, g=0)+n(R, g=1)+n(R, g=\infty)
$$

and $d(>0)$ is the minimum distance from the origin to all the points of $g(\varsigma)=0,1$ or $\infty$, then

$$
\begin{aligned}
T(r, g)< & \frac{C R \mathfrak{N}}{R-r}\left\{\log \frac{R \mathfrak{N}}{d}+\log ^{+} R+\log ^{+} \frac{1}{R}+\log \frac{2 R}{R-r}\right\} \\
& +\log ^{+}|g(0)|
\end{aligned}
$$

for $0<r<R$, where $C$ is a numerical constant.

\section{Property of functions having deficient values.}

4.1. We need more preparations to prove the main result formulated in the introduction.

LEMMA 2. Let $f(z)$ be an entire function of lower order $\mu$, where $0<\mu<+\infty$. If $f(z)$ has $p_{0}\left(0 \leq p_{0}<+\infty\right)$ finite deficient values $a_{j}\left(j=1,2, \ldots, p_{0}\right), f^{(-1)}(z)$ has $p_{-1}\left(0 \leq p_{-1}<+\infty\right)$ finite deficient values $b_{l}\left(l=1,2, \ldots, p_{-1}\right)$ and

$$
\delta=\min _{\substack{1 \leq j \leq p_{0} \\ 1 \leq l \leq p_{-1}}}\left\{\delta\left(a_{j}, f\right), \delta\left(b_{l}, f^{(-1)}\right)\right\},
$$

then there exists a sequence $\left\{R_{k}\right\}$ of positive numbers such that

$$
\begin{gathered}
t_{k} \leq R_{k} \leq 2 t_{k} \\
\operatorname{mes} E_{k j}=\operatorname{mes} E\left\{\theta: \log \left|f\left(R_{k} e^{i \theta}\right)-a_{j}\right| \leq-(\delta / 4) T\left(R_{k}, f\right)\right\}>B \\
\left(j=1,2, \ldots, p_{0}\right)
\end{gathered}
$$

and

(4.3) $\operatorname{mes} E_{k l}^{(-1)}=\operatorname{mes} E\left\{\theta: \log \left|f^{(-1)}\left(R_{k} e^{i \theta}\right)-b_{l}\right| \leq-(\delta / 4) T\left(R_{k}, f\right)\right\}>B$

$$
\left(l=1,2, \ldots, p_{-1}\right)
$$

where $k$ is sufficiently large. The sequence $\left\{t_{k}\right\}$ is determined by Lemma 1 with $\beta_{1}=8$ and $\beta_{2}=4 \cdot 10^{4 V}(V$ will be given by (6.6)) and

$$
B=B\left(\delta, \mu, p_{0}, p_{-1}\right)=\frac{\delta \pi}{6 \cdot 8^{\mu}\left(6+\log \left\{40\left(p_{0}+p_{-1}\right) e\right\} / \log \frac{4}{3}\right)}>0 .
$$


PrOOF. According to Lemma 1 , there is a sequence $\left\{t_{k}\right\}$ satisfying

$$
\begin{gathered}
\lim _{k \rightarrow+\infty} \frac{\log T\left(t_{k}, f\right)}{\log t_{k}}=\mu, \\
T\left(8 t_{k}, f\right)<\left(8^{\mu}+o(1)\right) T\left(t_{k}, f\right)
\end{gathered}
$$

and

$$
T\left(4 \cdot 10^{4 V} t_{k}, f\right)<\left\{\left(8 \cdot 4 \cdot 10^{4 V}\right)^{\mu}+o(1)\right\} T\left(t_{k}, f\right) .
$$

Let $k$ be sufficiently large and fixed. In $|z| \leq 3 t_{k}$, denote by $\alpha_{j s}\left(s=1,2, \ldots, n_{j}\right.$; $\left.n_{j}=n\left(3 t_{k}, f=a_{j}\right)\right)$ the zeros of $f(z)-a_{j}$ and by $\beta_{l u}\left(u=1,2, \ldots, n_{l}^{(-1)} ; n_{l}^{(-1)}=\right.$ $\left.n\left(3 t_{k}, f^{(-1)}=b_{l}\right)\right)$ the zeros of $f^{(-1)}(z)-b_{l}$. From the Boutroux-Cartan theorem [9], we have

$$
\prod_{s=1}^{n_{j}}\left|z-\alpha_{j s}\right|>H^{n_{j}} \quad\left(j=1,2, \ldots, p_{0}\right)
$$

and

$$
\prod_{u=1}^{n_{l}^{(-1)}}\left|z-\beta_{l u}\right|>H^{n_{l}^{(-1)}} \quad\left(l=1,2, \ldots, p_{-1}\right)
$$

except a set which can be enclosed in a finite number of disks with the sum of total radii not exceeding $2 e\left(p_{0}+p_{-1}\right) H$. The union of these disks is denoted by $(\gamma)$.

Choose

$$
H=t_{k} / 8 e\left(p_{0}+p_{-1}\right) \text {. }
$$

There exists a positive number $R_{k}$ such that

$$
R_{k} \in\left[t_{k}, 2 t_{k}\right], \quad\left(|z|=R_{k}\right) \cap(\gamma)=\varnothing .
$$

Since $R_{k} e^{i \theta} \notin(\gamma)(0 \leq \theta<2 \pi),(4.7),(4.9)$ and (4.6), we obtain

$$
\begin{aligned}
\log \frac{1}{\left|f\left(R_{k} e^{i \theta}\right)-a_{j}\right|} \leq & \frac{3 t_{k}+R_{k}}{3 t_{k}-R_{k}} m\left(3 t_{k}, \frac{1}{f-a_{j}}\right) \\
& +\sum_{s=1}^{n_{j}} \log \left|\frac{\left(3 t_{k}\right)^{2}-\bar{\alpha}_{j s} R_{k} e^{i \theta}}{3 t_{k}\left(R_{k} e^{i \theta}-\alpha_{j s}\right)}\right| \\
\leq & 5 m\left(3 t_{k}, \frac{1}{f-a_{j}}\right)+n_{j}\left(\log 5 t_{k}+\log \frac{1}{H}\right) \\
\leq & 5 m\left(3 t_{k}, \frac{1}{f-a_{j}}\right)+\frac{N\left(4 t_{k}, f=a_{j}\right)}{\log \frac{4}{3}} \log \left\{40\left(p_{0}+p_{-1}\right) e\right\} \\
& <\left\{5+\frac{\log \left\{40\left(p_{0}+p_{-1}\right) e\right\}}{\log \frac{4}{3}}+o(1)\right\} T\left(8 t_{k}, f\right) \\
& <8^{\mu}\left\{5+\frac{\log \left\{40\left(p_{0}+p_{-1}\right) e\right\}}{\log \frac{4}{3}}+o(1)\right\} T\left(t_{k}, f\right) .
\end{aligned}
$$


On the other hand, we have

$$
\begin{aligned}
\frac{\delta}{2} T\left(R_{k}, f\right) & <\frac{1}{2 \pi} \int_{0}^{2 \pi} \log ^{+} \frac{1}{\left|f\left(R_{k} e^{i \theta}\right)-a_{j}\right|} d \theta \\
& <\frac{1}{2 \pi} \int_{E_{k j}} \log ^{+} \frac{1}{\left|f\left(R_{k} e^{i \theta}\right)-a_{j}\right|} d \theta+\frac{\delta}{4} T\left(R_{k}, f\right) .
\end{aligned}
$$

Substituting (4.10) into this inequality, we obtain

$$
\begin{aligned}
\frac{\delta}{4} T\left(R_{k}, f\right) & <\frac{1}{2 \pi} \int_{E_{k j}} \log ^{+} \frac{1}{\left|f\left(R_{k} e^{i \theta}\right)-a_{j}\right|} d \theta \\
& <\frac{1}{2 \pi} 8^{\mu}\left\{5+\frac{\log \left\{40\left(p_{0}+p_{-1}\right) e\right\}}{\log \frac{4}{3}}+o(1)\right\} T\left(R_{k}, f\right) \operatorname{mes} E_{k j} .
\end{aligned}
$$

Therefore (4.2) is proved.

Similarly we obtain

$$
\log \frac{1}{\left|f^{(-1)}\left(R_{k} e^{i \theta}\right)-b\right|}<\left\{5+\frac{\log \left\{40\left(p_{0}+p_{-1}\right) e\right\}}{\log \frac{4}{3}}+o(1)\right\} T\left(4 t_{k}, f^{(-1)}\right) .
$$

For sufficiently large $r$, from

$$
\begin{aligned}
T\left(r, f^{(-1)}\right) & \leq \log M\left(r, f^{(-1)}\right) \\
& \leq \log \left\{\left|f^{(-1)}(0)\right|+r M(r, f)\right\} \\
& <(3+o(1)) T(2 r, f)
\end{aligned}
$$

and (4.6), we have

$$
T\left(4 t_{k}, f^{(-1)}\right)<(3+o(1)) T\left(8 t_{k}, f\right)<\left(3 \cdot 8^{\mu}+o(1)\right) T\left(t_{k}, f\right) .
$$

Hence

$$
\log \frac{1}{\left|f^{(-1)}\left(R_{k} e^{i \theta}\right)-b_{l}\right|}<3 \cdot 8^{\mu}\left\{5+\frac{\log \left\{40\left(p_{0}+p_{-1}\right) e\right\}}{\log \frac{4}{3}}+o(1)\right\} T\left(t_{k}, f\right) .
$$

The same procedure yields

$$
\begin{aligned}
& \frac{\delta}{4} T\left(R_{k}, f^{(-1)}\right) \\
& \quad<\frac{1}{2 \pi} 3 \cdot 8^{\mu}\left\{5+\frac{\log \left\{40\left(p_{0}+p_{-1}\right) e\right\}}{\log \frac{4}{3}}+o(1)\right\} T\left(R_{k}, f\right) \operatorname{mes} E_{k l}^{(-1)}
\end{aligned}
$$

Since

$$
T\left(R_{k}, f\right) \leq T\left(R_{k}, f^{(-1)}\right)+m\left(R_{k}, f / f^{(-1)}\right)
$$

and

$$
m\left(R_{k}, \frac{f}{f^{(-1)}}\right)<O(1)+4 \log ^{+} \rho+3 \log ^{+} \frac{1}{\rho-R_{k}}+4 \log ^{+} T\left(\rho, f^{(-1)}\right)
$$

$$
\left(R_{k}<\rho\right)
$$

we have

$$
\begin{aligned}
\log ^{+} T\left(\rho, f^{(-1)}\right) & <\log ^{+}\left\{4 T\left(8 t_{k}, f\right)\right\} \\
& <\log ^{+}\left\{4\left(8^{\mu}+o(1)\right) T\left(t_{k}, f\right)\right\}=O\left(\log R_{k}\right)
\end{aligned}
$$


by choosing $\rho=4 t_{k},(4.11),(4.6)$ and (4.5). Thus

$$
(\delta / 4-o(1)) T\left(R_{k}, f\right)<(\delta / 4) T\left(R_{k}, f^{(-1)}\right) .
$$

Comparing (4.12) with (4.13), we derive (4.3).

REMARK. We may replace $f^{(-1)}(z)$ by $f^{(l)}(z)(-1 \geq l \geq-L)$ and obtain a similar result.

\section{Properties of functions having no Borel directions in an angle.}

5.1. Now we shall establish a property of meromorphic functions without Borel directions in an angle.

LEMMA 3. Let $f(z)$ be an entire function of lower order $\mu$ and order $\lambda$, where $0<\mu \leq \lambda<+\infty$. Suppose that $f(z)$ has no Borel direction of order $\geq \mu$ in the angle $G: \theta_{1}<\arg z<\theta_{2}$. If there are positive numbers $\delta$ and $B$, a finite complex value $a_{0}$ and a positive and sufficiently large number ${ }^{2} R_{k}$ such that

$$
\text { mes } E\left\{\theta: \theta_{1}<\theta<\theta_{2}, \log \left|f\left(R_{k} e^{i \theta}\right)-a_{0}\right| \leq-(\delta / 4) T\left(R_{k}, f\right)\right\}>B,
$$

then for two positive numbers $\alpha$ and $Q, 0<\alpha<\frac{1}{4}\left(\theta_{2}-\theta_{1}\right), Q>1$, we have

$$
\log \left|f(z)-a_{0}\right|<-C_{0} T\left(R_{k}, f\right)
$$

and

$$
\log \left|f^{(j)}(z)\right|<-C_{0} T\left(R_{k}, f\right)+\log \left\{j !\left(\frac{20 \cdot 10^{4 Q}}{\alpha R_{k}}\right)^{j}\right\} \quad(j=1,2, \ldots)
$$

in the region

$$
D_{k}:\left(10^{-4 Q} R_{k} \leq|z| \leq 10^{4 Q} R_{k}\right) \cap\left(\theta_{1}+\alpha \leq \arg z \leq \theta_{2}-\alpha\right),
$$

where

and

$$
\begin{aligned}
C_{0}=\frac{\delta}{4\left\{4\left(5+4 \log \frac{1}{h}\right)\right\}^{2 N_{1}+N_{2}}}, & \\
& N_{1}=\left[\frac{10 \pi}{\alpha}\right], \quad N_{2}=\left[\frac{20\left(10^{4 Q}-1\right)}{\alpha}\right]+4,
\end{aligned}
$$

$$
\operatorname{mes}\left\{\varphi: \theta_{1}+\frac{4}{5} \alpha \leq \varphi \leq \theta_{2}-\frac{4}{5} \alpha, \log \left|f\left(R_{k} e^{i \varphi}\right)-a_{0}\right| \leq-\frac{\delta}{4} T\left(R_{k}, f\right)\right\}>\frac{B}{2} \text {. }
$$

We divide $G$ into $N_{1}$ equal angles $G_{\nu}\left(\nu=1,2, \ldots, N_{1} ; N_{1}=[10 \pi / \alpha]+1\right)$, so the opening of $G_{\nu}$ does not exceed $\alpha / 5$. There is an angle $G_{\nu_{0}}$ such that

$$
\begin{aligned}
\operatorname{mes} & \left\{\varphi: R_{k} e^{i \varphi} \in G_{\nu_{0}}, \log \left|f\left(R_{k} e^{i \varphi}\right)-a_{0}\right| \leq-\frac{\delta}{4} T\left(R_{k}, f\right)\right\} \\
& >\frac{B}{2 N_{1}} \geq \frac{\alpha B}{2(10 \pi+\alpha)} .
\end{aligned}
$$

\footnotetext{
${ }^{2}$ In the late use of $\S 7$, there is a sequence of positive numbers which tend to infinity and satisfy (5.1) for every $k$. For the abbreviation of notations, we consider only one sufficiently large number $R_{k}$ here.
} 
Since $f(z)$ has no Borel direction of order $\geq \mu$ in $G$, two finite distinct complex values $\beta_{l}(l=1,2)$ and a positive number $\tau<\mu$ can be found with

$$
\sum_{l=1}^{2} n\left\{r, \theta_{1}+\frac{\alpha}{10}, \theta_{2}-\frac{\alpha}{10}, f=\beta_{l}\right\}<r^{\tau}
$$

by Lemma A, provided that $r$ is sufficiently large.

Write

$$
\begin{aligned}
n_{1}= & \sum_{l=1}^{2} n\left(\left(1+\frac{3}{5} \alpha\right) R_{k}, \theta_{1}+\frac{\alpha}{10}, \theta_{2}-\frac{\alpha}{10}, f=\beta_{l}\right) \\
& +n\left(\left(1+\frac{3}{5} \alpha\right) R_{k}, \theta_{1}+\frac{\alpha}{10}, \theta_{2}-\frac{\alpha}{10}, f=a_{0}\right) .
\end{aligned}
$$

Then we construct some disks, having their centres at every zero of $f(z)-\beta_{l}$ $(l=1,2)$ and of $f(z)-a_{0}$ in the region $\left(|z| \leq\left(1+\frac{3}{5} \alpha\right) R_{k}\right) \cap\left(\theta_{1}+\alpha / 10 \leq \arg z \leq\right.$ $\left.\theta_{2}-\alpha / 10\right)$ and having the same radius $\alpha h R_{k} / 2\left(n_{1}+1\right)$, where $h$ is given by (5.6). The union of these disks is denoted by $(\gamma)_{1}$.

Let $z_{1}$ be the point of intersection of $|z|=R_{k}$ and the bisector of $G_{\nu_{0}}$ and let

$$
\Gamma_{k}:\left|z-z_{1}\right|<\alpha R_{k} / 10 \text {. }
$$

Denote by $d_{s}\left(s=1,2, \ldots, n_{2}\right)$ the zeros of $f(z)-a_{0}$ in $\left|z-z_{2}\right|<\frac{3}{10} \alpha R_{k}$, where $z_{2}$ is an arbitrary point in $\Gamma_{k} \backslash(\gamma)_{1}$. According to the Boutroux-Cartan theorem, we have

$$
\prod_{s=1}^{n_{2}}\left|z-d_{s}\right|>\left(\frac{h \alpha R_{k}}{2}\right)^{n_{2}}
$$

except a set contained in several disks with the sum of radii not exceeding $e h \alpha R_{k}$. Denote by $(\gamma)_{2}$ the union of these disks.

Since the total sum of diameters of $(\gamma)_{1}$ and $(\gamma)_{2}$ does not exceed

$$
\alpha h R_{k}+2 e h \alpha R_{k}=\frac{\alpha^{2} K}{8(10 \pi+\alpha)} R_{k},
$$

there is a point $z_{3}$ in $\Gamma_{k} \backslash\left((\gamma)_{1} \cup(\gamma)_{2}\right)$ such that

$$
\log \left|f\left(z_{3}\right)-a_{0}\right|<-(\delta / 4) T\left(R_{k}, f\right) .
$$

Applying the Poisson-Jensen's formula [5], we have

$$
\begin{aligned}
\log \frac{1}{\left|f\left(z_{3}\right)-a_{0}\right|} & \leq 5 m\left(\frac{3}{10} \alpha R_{k}, z_{2}, \frac{1}{f-a_{0}}\right)+\sum \log \left|\frac{\left(\frac{3}{10} \alpha R_{k}\right)^{2}-\bar{d}_{s} z_{3}}{\frac{3}{10} \alpha R_{k}\left(z_{3}-d_{s}\right)}\right| \\
& <\left(5+4 \log \frac{1}{h}\right) T\left(\frac{2}{5} \alpha R_{k}, z_{2}, \frac{1}{f-a_{0}}\right),
\end{aligned}
$$

where

$$
m\left(\frac{3}{10} \alpha R_{k}, z_{2}, 1 /\left(f-a_{0}\right)\right) \text { and } T\left(\frac{2}{5} \alpha R_{k}, z_{2}, 1 /\left(f-a_{0}\right)\right)
$$

denote

$$
m\left(\frac{3}{10} \alpha R_{k}, 1 /\left(f\left(z+z_{2}\right)-a_{0}\right)\right) \text { and } T\left(\frac{2}{5} \alpha R_{k}, 1 /\left(f\left(z+z_{2}\right)-a_{0}\right)\right)
$$


respectively. Further Lemma $\mathrm{C}$ gives

$$
\begin{aligned}
& T\left(\frac{2}{5} \alpha R_{k}, z_{2}, \frac{1}{f-a_{0}}\right) \\
& \leq T\left(\frac{2}{5} \alpha R_{k}, z_{2}, \frac{1 /\left(f-a_{0}\right)}{1 /\left(f-a_{0}\right)-1 /\left(\beta_{2}-a_{0}\right)} \cdot \frac{1 /\left(\beta_{1}-a_{0}\right)-1 /\left(\beta_{c_{2}}-a_{0}\right)}{1 /\left(\beta_{1}-a_{0}\right)}\right) \\
&+\log \left|\frac{1}{f\left(z_{2}\right)-a_{0}}-\frac{1}{\beta_{2}-a_{0}}\right|+C \\
&< C\left(\sum_{l=1}^{2} n\left(\frac{1}{2} \alpha R_{k}, z_{2}, f=\beta_{l}\right)\right) \\
& \times\left\{\log \frac{\sum_{l=1}^{2} n\left(\frac{1}{2} \alpha R_{k}, z_{2}, f=\beta_{l}\right)+1}{h /\left(n_{1}+1\right)}+\log R_{k}+\log 10\right\} \\
&+2 \log +\frac{1}{\left|f\left(z_{2}\right)-a_{0}\right|}+C,
\end{aligned}
$$

where $C$ is a numerical constant which need not be the same at each occurrence.

In view of

$$
\sum_{l=1}^{2} n\left(\frac{1}{2} \alpha R_{k}, z_{2}, f=\beta_{l}\right)=O\left(R_{k}^{\tau}\right)
$$

and $n_{1}<R_{k}^{\lambda+1}$, we obtain from (5.8), (5.9) and (5.10) that

$$
\frac{\delta}{8} T\left(R_{k}, f\right)<2\left(5+4 \log \frac{1}{h}\right) \log \frac{1}{\left|f\left(z_{2}\right)-a_{0}\right|},
$$

i.e.

$$
\log \frac{1}{\left|f\left(z_{2}\right)-a_{0}\right|}>\frac{\delta}{16\left(5+4 \log \frac{1}{h}\right)} T\left(R_{k}, f\right) .
$$

We rotate $\Gamma_{k}$ about the origin $\alpha / 10$ in succession. Then a chain $\Gamma_{\Sigma}$ of $2 N_{1}$ disks at most can be obtained to cover the region

$$
\left(R_{k}-\frac{\sqrt{3}}{20} \alpha R_{k} \leq|z| \leq R_{k}+\frac{\sqrt{3}}{20} \alpha R_{k}\right) \cap\left(\theta_{1}+\frac{4}{5} \alpha \leq \arg z \leq \theta_{2}-\frac{4}{5} \alpha\right) .
$$

By the same procedure, we can prove that

$$
\left|f(z)-a_{0}\right|<\exp \left(-\frac{\delta}{4\left\{4\left(5+4 \log \frac{1}{h}\right)\right\}^{2 N_{1}}} T\left(R_{k}, f\right)\right)
$$

for any point $z \in\left(\Gamma_{\Sigma} \backslash(\gamma)_{1}\right)$. Since the total sum of diameters of disks in $(\gamma)_{1}$ does not exceed $\alpha h R_{k}<\alpha R_{k} / 80$, (5.11) holds without exception in the region

$$
\left(R_{k}-\frac{1}{20} \alpha R_{k} \leq|z| \leq R_{k}+\frac{1}{20} \alpha R_{k}\right) \cap\left(\theta_{1}+\frac{33}{40} \alpha \leq \arg z \leq \theta_{2}-\frac{33}{40} \alpha\right)
$$

by the maximum modulus theorem.

Let $z_{0}=R_{k} e^{i \theta_{0}}$ be an arbitrary point on

$$
\left(|z|=R_{k}\right) \cap\left(\theta_{1}+\frac{33}{40} \alpha \leq \arg z \leq \theta_{2}-\frac{33}{40} \alpha\right) .
$$


We make two concentric disks

$$
\Gamma_{k}^{\prime}:\left|z-z_{0}\right| \leq \frac{1}{20} \alpha R_{k}, \quad \Gamma_{k}^{\prime \prime}:\left|z-z_{0}\right| \leq \frac{\alpha R_{k}}{20 \cdot 10^{4 Q}}
$$

where $Q>1$ is mentioned in the lemma. With the centre of $\Gamma_{k}^{\prime}$ on the segment

$$
L_{1}:\left\{r e^{i \theta_{0}}: R_{k} \leq r \leq\left(10^{4 Q}+\frac{\alpha}{10}\right) R_{k}\right\},
$$

we move $\Gamma_{k}^{\prime}$ along $L_{1}$ by distance $\alpha R_{k} / 20$ each time. Then $L_{1}$ is covered by $N_{2}=\left[\left(20 \cdot 10^{4 Q}\right) / \alpha\right]+4$ at most of these disks. Similarly, we move $\Gamma_{k}^{\prime \prime}$ by distance $\alpha R_{k} /\left(20 \cdot 10^{4 Q}\right)$ each time along

$$
L_{2}:\left\{\left(1 / 10^{4 Q}-\alpha /\left(5 \cdot 10^{4 Q}\right)\right) R_{k} \leq r \leq R_{k}\right\}
$$

and $L_{2}$ is covered by at most $N_{2}$ of these disks.

Therefore we have (5.2) in the region

$$
\begin{gathered}
\left\{\left(\frac{1}{10^{4 Q}}-\frac{\alpha}{5 \cdot 10^{4 Q}}\right) R_{k} \leq|z| \leq\left(10^{4 Q}+\frac{\alpha}{10}\right) R_{k}\right\} \\
\cap\left(\theta_{1}+\frac{9}{10} \alpha \leq \arg z \leq \theta_{2}-\frac{9}{10} \alpha\right),
\end{gathered}
$$

hence (5.3) by the Cauchy inequality.

5.2. We establish a corresponding lemma for the primitive.

LEMMA 4. Let $f(z)$ be an entire function of lower order $\mu$ and order $\lambda$, where $0<\mu \leq \lambda<+\infty$. Suppose that $f(z)$ has no Borel direction of order $\geq \mu$ in the angle $\theta_{1}<\arg z<\theta_{2}$. If there are two positive numbers $\delta$ and $B$, a finite complex value $b_{0}$ and a sufficiently large positive number $R_{k}$ such that

$$
\begin{aligned}
& \operatorname{mes} E_{k}^{(-1)}=\operatorname{mes} E\left\{\theta: \theta_{1}<\theta<\theta_{2}, \log \left|f^{(-1)}\left(R_{k} e^{i \theta}\right)-b_{0}\right|\right. \\
&\left.\leq-(\delta / 4) T\left(R_{k}, f\right)\right\}>B
\end{aligned}
$$

then we have in $D_{k}$ defined by (5.4)

$$
\begin{gathered}
\log \left|f^{(-1)}(z)-b_{0}\right|<-(\delta / 8) T\left(R_{k}, f\right)<-C_{0} T\left(R_{k}, f\right), \\
\log |f(z)|<-\left(C_{0} / 2\right) T\left(R_{k}, f\right)
\end{gathered}
$$

and

$$
\log \left|f^{(j)}(z)\right|<-\frac{C_{0}}{2} T\left(R_{k}, f\right)+\log \left\{j !\left(\frac{20 \cdot 10^{4 Q}}{\alpha R_{k}}\right)^{j}\right\}, \quad j=1,2, \ldots,
$$

where $C_{0}$ is given by (5.5).

PrOOF. Write

$$
N=n\left(2 R_{k}, f^{(-1)}=b_{0}\right)+n\left(2 R_{k}, f=0\right)
$$

and $d=B R_{k} / 4 N$. Let $(\gamma)$ be the union of disks, having centres at zeros of $f^{(-1)}(z)-b_{0}$ and of $f(z)$ in $|z| \leq 2 R_{k}$ and having the same radius $d$. Since the total sum of radii does not exceed $N d=B R_{k} / 4$, we have

$$
\operatorname{mes} E_{k}^{(-1)^{*}}=\operatorname{mes} E\left\{\theta: \theta \in E_{k}^{(-1)}, R_{k} e^{i \theta} \bar{\varepsilon}(\gamma)\right\}>B / 2 \text {. }
$$


When $\theta \in E_{k}^{(-1)^{*}}$, differentiation of the Poisson-Jensen formula gives

$$
\begin{aligned}
\left|\frac{f\left(R_{k} e^{i \theta}\right)}{f^{(-1)}\left(R_{k} e^{i \theta}\right)-b_{0}}\right| \leq & \frac{4}{R_{k}} \cdot \frac{1}{2 \pi} \int_{0}^{2 \pi} \log ^{+}\left|f^{(-1)}\left(2 R_{k} e^{i \varphi}\right)-b_{0}\right| d \varphi \\
& +\sum \frac{\left(2 R_{k}\right)^{2}}{2 R_{k}^{2}\left|R_{k} e^{i \theta}-\beta_{\nu}\right|},
\end{aligned}
$$

where $\beta_{\nu}$ are zeros of $f^{(-1)}(z)-b_{0}$ in $|z| \leq 2 R_{k}$. Thus

$$
\begin{aligned}
\log \left|f\left(R_{k} e^{i \theta}\right)\right| \leq & \log \left|f^{(-1)}\left(R_{k} e^{i \theta}\right)-b_{0}\right| \\
& +\log ^{+} m\left(2 R_{k}, f^{(-1)}-b_{0}\right)+\log ^{+} m\left(2 R_{k}, \frac{1}{f^{(-1)}-b_{0}}\right) \\
& +\log ^{+} \frac{1}{R_{k}}+\log ^{+} \frac{1}{d}+\log ^{+} n\left(2 R_{k}, f^{(-1)}=b_{0}\right)+5 \log 2 .
\end{aligned}
$$

Since the order of $f(z)$ equals $\lambda$, so does that of $f^{(-1)}(z)$. When $R_{k}$ is sufficiently large, we have

$$
\begin{aligned}
& \log ^{+} m\left(2 R_{k}, f^{(-1)}-b_{0}\right)=O\left(\log R_{k}\right), \\
& \log ^{+} m\left(2 R_{k}, \frac{1}{f^{(-1)}-b_{0}}\right)=O\left(\log R_{k}\right), \\
& \log ^{+} \frac{1}{d}=O\left(\log R_{k}\right)
\end{aligned}
$$

and

$$
\log ^{+} n\left(2 R_{k}, f^{(-1)}=b_{0}\right)=O\left(\log R_{k}\right) .
$$

In view of these estimates and $\mu>0$, we obtain

$$
\log \left|f\left(R_{k} e^{i \theta}\right)\right|<-(\delta / 8) T\left(R_{k}, f\right)
$$

for $\theta \in E_{k}^{(-1)^{*}}$.

Applying Lemma 3, it follows from (5.16) and (5.17) that

$$
\log |f(z)|<-\left(C_{0} / 2\right) T\left(R_{k}, f\right)
$$

and

$$
\log \left|f^{(j)}(z)\right|<-\frac{C_{0}}{2} T\left(R_{k}, f\right)+\log \left\{j !\left(\frac{20 \cdot 10^{4 Q}}{\alpha R_{k}}\right)^{j}\right\}
$$

in the region $D_{k}$ defined by (5.4).

When $\theta_{0} \in E_{k}^{(-1)}$ and $z_{0}=R_{k} e^{i \theta_{0}}$, we have

$$
\left|f^{(-1)}(z)-b_{0}\right| \leq\left|f^{(-1)}\left(z_{0}\right)-b_{0}\right|+\left|\int_{\overline{z_{0} z}} f(\varsigma) d \varsigma\right|
$$

for $z \in D_{k}$. Thus (5.13) follows immediately from (5.12), (5.14) and (5.18).

REMARK. Replacing $f^{(-1)}(z)$ by $f^{(l)}(z)$, where $l$ is a negative integer, we can obtain a similar lemma. 


\section{Estimation of harmonic measure.}

6.1. We need some estimates of harmonic measure.

LEMMA 5. Let $\alpha$ and $Q$ be two numbers with $0<\alpha<\pi / 6$ and $Q>1$. Putting

$$
D:\left(10^{-2 Q} R_{k}<|z|<10^{2 Q} R_{k}\right) \cap(\theta-\alpha<\arg z<\theta+5 \alpha)
$$

and

$$
\Gamma_{1}:\left(10^{-2 Q} R_{k}<|z|<10^{2 Q} R_{k}\right) \cap(\arg z=\theta-\alpha),
$$

then $\omega\left(z_{0}, \Gamma_{1} ; D\right) \geq \frac{1}{4}$ for any point $z_{0}$ on the arc $\left(|z|=R_{k}\right) \cap(\theta-\alpha<\arg z<$ $\theta+2 \alpha)$.

PROOF. Write

$$
\begin{aligned}
& \Gamma_{2}:\left(10^{-2 Q} R_{k}<|z|<10^{2 Q} R_{k}\right) \cap(\arg z=\theta+5 \alpha), \\
& \Gamma_{3}:\left(|z|=10^{-2 Q} R_{k}\right) \cap(\theta-\alpha<\arg z<\theta+5 \alpha)
\end{aligned}
$$

and

$$
\Gamma_{4}:\left(|z|=10^{2 Q} R_{k}\right) \cap(\theta-\alpha<\arg z<\theta+5 \alpha) .
$$

It is clear that

$$
\sum_{j=1}^{4} \omega\left(z_{0}, \Gamma_{j} ; D\right)=1
$$

Further, denoting $D_{1}: \theta-\alpha<\arg z<\theta+5 \alpha$ and $\Gamma_{2}^{\prime}: \arg z=\theta+5 \alpha$, we have

$$
\begin{aligned}
\omega\left(z_{0}, \Gamma_{2} ; D\right) & \leq \omega\left(z_{0}, \Gamma_{2} ; D_{1}\right) \leq \omega\left(z_{0}, \Gamma_{2}^{\prime} ; D_{1}\right) \\
& =\frac{\theta_{0}-(\theta-\alpha)}{(\theta+5 \alpha)-(\theta-\alpha)} \leq \frac{1}{2},
\end{aligned}
$$

where $\theta_{0}=\arg z_{0}$. From this estimate of harmonic measure, we know that

$$
\omega\left(z_{0}, \Gamma_{3} ; D\right) \leq \frac{4\left(\left(10^{-2 Q} R_{k}\right) /\left|z_{0}\right|\right)^{\pi / 6 \alpha}}{\pi\left\{1-\left(10^{-2 Q} R_{k}\right) /\left|z_{0}\right|\right\}^{2 \pi / 6 \alpha}}<\frac{1}{8}
$$

and

$$
\omega\left(z_{0}, \Gamma_{4} ; D\right)<\frac{1}{8}
$$

Substituting (6.2), (6.3) and (6.4) into (6.1), we derive the conclusion of Lemma 5.

6.2. LEMMA 6. Let $\alpha$ and $Q$ be two numbers with $0<\alpha<\pi / 12$ and $Q>1$. Denoting

$$
D:\left(10^{-4 Q} R_{k}<|z|<10^{4 Q} R_{k}\right) \cap(\theta-\alpha<\arg z<\theta+11 \alpha)
$$

and

$$
\Gamma_{1}:\left(10^{-4 Q} R_{k}<|z|<10^{4 Q} R_{k}\right) \cap(\arg z=\theta-\alpha),
$$

we have $\omega\left(z_{0}, \Gamma_{1} ; D\right) \geq \frac{1}{4}$ for any point in the region

$$
\left(10^{-2 Q} R_{k}<|z|<10^{2 Q} R_{k}\right) \cap(\theta-\alpha<\arg z<\theta+5 \alpha) .
$$


PROOF. In a similar way to the proof of Lemma 5, we write

$$
\begin{aligned}
& \Gamma_{2}:\left(10^{-4 Q} R_{k}<|z|<10^{4 Q} R_{k}\right) \cap(\arg z=\theta+11 \alpha), \\
& \Gamma_{3}:\left(|z|=10^{-4 Q} R_{k}\right) \cap(\theta-\alpha<\arg z<\theta+11 \alpha), \\
& \Gamma_{4}:\left(|z|=10^{4 Q} R_{k}\right) \cap(\theta-\alpha<\arg z<\theta+11 \alpha), \\
& D_{1}: \theta-\alpha<\arg z<\theta+11 \alpha
\end{aligned}
$$

and $\Gamma_{2}^{\prime}: \arg z=\theta+11 \alpha$. Thus we have

$$
\omega\left(z_{0}, \Gamma_{2} ; D\right) \leq \omega\left(z_{0}, \Gamma_{2}^{\prime} ; D_{1}\right) \leq \frac{1}{2}, \quad \omega\left(z_{0}, \Gamma_{3} ; D\right)<\frac{1}{8}
$$

and $\omega\left(z_{0}, \Gamma_{4} ; D\right)<\frac{1}{8}$. Lemma 6 follows from these estimates and the fact that $\sum_{j=1}^{4} \omega\left(z_{0}, \Gamma_{j} ; D\right)=1$.

6.3. Finally we give an estimation between the maximum modulus and characteristic function.

LEMMA 7. Suppose that $f(z)$ satisfies the assumptions of Lemma 2 with $p_{0}+$ $p_{-1} \geq 1$. Then a positive number $V$ can be chosen sufficiently large such that

$$
\begin{aligned}
\max & \left\{\log M\left(10^{-4 V} R_{k}, f\right), \log M\left(10^{-4 V} R_{k}, f^{(-1)}\right)\right\} \\
& <\frac{1}{4}\left(C_{0} / 4\right)^{q+2} T\left(R_{k}, f\right),
\end{aligned}
$$

where $\left\{R_{k}\right\}$ is defined in Lemma 2, q denotes the number of Borel directions of $f(z)$ and $C_{0}$ is given by (5.5).

PROOF. When $f(z)$ has a finite deficient value $a_{1}$, according to a result of $\mathrm{A}$. Weitsman [11], there is a positive number $\tau=\tau\left(\delta\left(a_{1}, f\right), \delta(\infty, f)\right)$ such that the inequality

$$
\varliminf_{t \rightarrow+\infty} \frac{T(\sigma t, f)}{T(t, f)} \geq \sigma^{\tau}
$$

holds for any $\sigma \geq 1$. When $p_{0}=0$ and $f^{(-1)}(z)$ has a finite deficient value $b_{1}$, then $\delta\left(b_{1}, f^{(-1)}\right) \leq \delta(0, f)$ and there is $\tau_{1}=\tau_{1}(\delta(0, f), \delta(\infty, f))>0$ with

$$
\varliminf_{t \rightarrow+\infty} \frac{T(\sigma t, f)}{T(t, f)} \geq \sigma^{\tau_{1}}
$$

Setting $\tau^{*}=\min \left(\tau, \tau_{1}\right)$, we have

$$
\log M\left(10^{-4 V} R_{k}, f\right) \leq 3 T\left(2 \cdot 10^{-4 V} R_{k}, f\right)<4\left(2 \cdot 10^{-4 V}\right)^{\tau^{*}} T\left(R_{k}, f\right) .
$$

Since

$$
\begin{aligned}
\log M\left(r, f^{(-1)}\right) & \leq \log \left|f^{(-1)}(0)\right|+\log 2 r+\log M(r, f) \\
& \leq O(\log r)+3 T(2 r, f),
\end{aligned}
$$

it is easy to see that

$$
\begin{aligned}
\log M\left(10^{-4 V} R_{k}, f^{(-1)}\right) & \leq O\left(\log R_{k}\right)+3 T\left(2 \cdot 10^{-4 V} R_{k}, f\right) \\
& <4\left(2 \cdot 10^{-4 V}\right)^{\tau^{*}} T\left(R_{k}, f\right) .
\end{aligned}
$$

Therefore, if we choose

$$
V>\frac{1}{4 \log 10}\left\{\left(1+\frac{4}{\tau^{*}}\right) \log 2+\frac{q+2}{\tau^{*}} \log \frac{4}{C_{0}}\right\},
$$

then (6.5) follows immediately. 
REMARK. In general, if $L$ is a negative integer, we can establish a similar lemma to prove that

$$
\max _{L \leq j \leq 0}\left\{\log M\left(10^{-4 V} R_{k}, f^{(j)}\right)\right\}<\frac{1}{4}\left(\frac{C_{0}}{4}\right)^{q+2} T\left(R_{k}, f\right) .
$$

\section{Proof of Theorem.}

7.1. Now we prove the theorem formulated in the introduction.

When $\mu \leq \frac{1}{2}$, the lower orders of $f^{(l)}(z)(l=0,-1,-2, \ldots)$ are all equal to $\mu$. Since an entire function of lower order less than or equal to $\frac{1}{2}$ cannot possess any finite deficient value, the conclusion of the theorem is clear.

When $\mu>\frac{1}{2}$, we note first by Lemma B that the order $\lambda$ of $f(z)$ must be finite, because the number of its Borel directions of order $\geq \mu$ is finite. For simplicity of notation, we only prove $p_{0}+p_{-1} \leq 2 \mu$. The same procedure can derive the general conclusion $\sum_{l=0}^{-\infty} p_{l} \leq 2 \mu$.

Suppose that $1 \leq p_{0}<+\infty$ and $1 \leq p_{-1}<+\infty$. Let $a_{j}\left(j=1,2, \ldots, p_{0}\right)$ be the deficient values of $f(z)$ with deficiencies $\delta\left(a_{j}, f\right), b_{l}\left(l=1,2, \ldots, p_{-1}\right)$ be the deficient values of $f^{(-1)}(z)$ with deficiencies $\delta\left(b_{l}, f^{(-1)}\right)$ and

$$
\delta=\min _{\substack{1 \leq j \leq p_{0} \\ 1 \leq l \leq p_{-1}}}\left\{\delta\left(a_{j}, f\right), \delta\left(b_{l}, f^{(-1)}\right)\right\} .
$$

From Lemma 2, there are two sequences $\left\{t_{k}\right\}$ and $\left\{R_{k}\right\}$ such that

$$
t_{k} \leq R_{k} \leq 2 t_{k}, \quad \lim _{k \rightarrow+\infty} \frac{\log T\left(t_{k}, f\right)}{\log t_{k}}=\mu,
$$

$\operatorname{mes} E\left\{\theta: \log \left|f\left(R_{k} e^{i \theta}\right)-a_{j}\right| \leq-(\delta / 4) T\left(R_{k}, f\right)\right\}>B, \quad j=1,2, \ldots, p_{0}$,

and

$$
\operatorname{mes} E\left\{\theta: \log \left|f^{(-1)}\left(R_{k} e^{i \theta}\right)-b_{l}\right| \leq-(\delta / 4) T\left(R_{k}, f\right)\right\}>B, \quad l=1,2, \ldots, p_{-1},
$$

where $B=B\left(\delta, \mu, p_{0}, p_{-1}\right)>0$ is determined by (4.4).

Denote by $\arg z=\theta_{\nu}\left(\nu=1,2, \ldots, q, 0 \leq \theta_{1}<\theta_{2}<\cdots<\theta_{q}<2 \pi\right)$ the $q$ Borel directions of order $\geq \mu$ of $f(z)$ which divide the plane into $q$ sectors. Let

$$
\omega=\min _{1 \leq \nu \leq q}\left(\theta_{\nu+1}-\theta_{\nu}\right), \quad \theta_{q+1}=2 \pi+\theta_{1} .
$$

Choose a fixed integer $k$, sufficiently large, so that corresponding to $a_{1}$ there is an angle, say $\theta_{\nu_{1}}<\arg z<\theta_{\nu_{1}+1}$, such that

$$
\operatorname{mes} E\left\{\theta: \theta_{\nu_{1}}<\theta<\theta_{\nu_{1}+1}, \log \left|f\left(R_{k} e^{i \theta}\right)-a_{1}\right| \leq-(\delta / 4) T\left(R_{k}, f\right)\right\}>B / q .
$$

Hereinafter we denote by $A\left(r_{1}, r_{2} ; \varphi_{1}, \varphi_{2}\right)$ the region $\left(r_{1}<|z|<r_{2}\right) \cap$ $\left(\varphi_{1}<\arg z<\varphi_{2}\right)$ and $\bar{A}\left(r_{1}, r_{2} ; \varphi_{1}, \varphi_{2}\right)$ its closure.

With two positive numbers $\alpha<\min (K / 4 q, \omega / 24)$ and $Q>1$ chosen, applying Lemma 3 to $f(z)$ and $a_{1}$ yields that

$$
\log \left|f(z)-a_{1}\right|<-C_{0} T\left(R_{k}, f\right)
$$

in the region $\bar{A}\left(10^{-4 Q} R_{k}, 10^{4 Q} R_{k} ; \theta_{\nu_{1}}+\alpha, \theta_{\nu_{1}+1}-\alpha\right)$, where $C_{0}$ is given by (5.5).

Similarly, corresponding to $a_{2}$ there is an angle, say $\theta_{\nu_{2}}<\arg z<\theta_{\nu_{2}+1}$, such that

$$
\operatorname{mes} E\left\{\theta: \theta_{\nu_{2}}<\theta<\theta_{\nu_{2}+1}, \log \left|f\left(R_{k} e^{i \theta}\right)-a_{2}\right| \leq-(\delta / 4) T\left(R_{k}, f\right)\right\}>B / q .
$$


Hence the inequality

$$
\log \left|f(z)-a_{2}\right|<-C_{0} T\left(R_{k}, f\right)
$$

holds in the region $\bar{A}\left(10^{-4 Q} R_{k}, 10^{4 Q} R_{k} ; \theta_{\nu_{2}}+\alpha, \theta_{\nu_{2}+1}-\alpha\right)$. It is easy to see that the angles $\theta_{\nu_{1}}<\arg z<\theta_{\nu_{1}+1}$ and $\theta_{\nu_{2}}<\arg z<\theta_{\nu_{2}+1}$ have no common point.

By this procedure, there are $p_{0}+p_{-1}$ angles, one corresponding to each of $a_{j}$ and $b_{l}, \theta_{\nu_{n}}<\arg z<\theta_{\nu_{n}+1}$

$$
0 \leq \theta_{\nu_{1}}<\theta_{\nu_{1}+1} \leq \theta_{\nu_{2}}<\theta_{\nu_{2}+1} \leq \cdots<\theta_{\nu_{p_{0}+p_{-1}}+1} \leq \theta_{1}+2 \pi,
$$

and in every angle $\theta_{\nu_{n}}<\arg z<\theta_{\nu_{n}+1}$, one an only one among the inequalities

$$
\log \left|f(z)-a_{j}\right|<-C_{0} T\left(R_{k}, f\right), \quad j=1,2, \ldots, p_{0},
$$

and

$$
\log \left|f^{(-1)}(z)-b_{l}\right|<-C_{0} T\left(R_{k}, f\right), \quad l=1,2, \ldots, p_{-1},
$$

holds uniformly in the region $\bar{A}\left(10^{-4 Q} R_{k}, 10^{4 Q} R_{k} ; \theta_{\nu_{n}}+\alpha, \theta_{\nu_{n}+1}-\alpha\right)$.

7.2. For two angles $\theta_{\nu_{1}}<\arg z<\theta_{\nu_{1}+1}$ and $\theta_{\nu_{2}}<\arg z<\theta_{\nu_{2}+1}$ corresponding to $a_{1}$ and $a_{2}$ respectively, we conclude that there is a point $z_{1}$, satisfying the condition $\log \left|f\left(z_{1}\right)-a_{1}\right|>0$ in the region $\bar{A}\left(10^{-2 Q} R_{k}, 10^{2 Q} R_{k} ; \theta_{\nu_{1}+1}-\alpha, \theta_{\nu_{2}}+5 \alpha\right)$.

In fact, if this assertion were not true, then the inequality

$$
\log \left|f(z)-a_{1}\right| \leq 0
$$

would hold uniformly in $\bar{A}\left(10^{-2 Q} R_{k}, 10^{2 Q} R_{k} ; \theta_{\nu_{1}+1}-\alpha, \theta_{\nu_{2}}+5 \alpha\right)$. Let

$$
\begin{gathered}
D_{k 1}: A\left(10^{-2 Q} R_{k}, 10^{2 Q} R_{k} ; \theta_{\nu_{1}+1}-\alpha, \theta_{\nu_{1}+1}+5 \alpha\right), \\
\Gamma_{1}:\left(10^{-2 Q} R_{k}<|z|<10^{2 Q} R_{k}\right) \cap\left(\arg z=\theta_{\nu_{1}+1}-\alpha\right)
\end{gathered}
$$

and $C \Gamma_{1}$ be the rest of the boundary of $D_{k 1}$. For an arbitrary point $z_{0}$ on the arc $\left(|z|=R_{k}\right) \cap\left(\theta_{\nu_{1}+1}-\alpha<\arg z<\theta_{\nu_{1}+1}+2 \alpha\right)$, we obtain

$$
\begin{aligned}
\log \left|f\left(z_{0}\right)-a_{1}\right| \leq & \omega\left(z_{0}, \Gamma_{1} ; D_{k 1}\right) \max _{z \in \Gamma_{1}} \log \left|f(z)-a_{1}\right| \\
& +\left\{1-\omega\left(z_{0}, \Gamma_{1} ; D_{k 1}\right)\right\} \max _{z \in C \Gamma_{1}} \log \left|f(z)-a_{1}\right| \\
< & -\left(C_{0} / 4\right) T\left(R_{k}, f\right),
\end{aligned}
$$

by noting $(7.1)$ on $\Gamma_{1},(7.5)$ and $\omega\left(z_{0}, \Gamma_{1} ; D_{k 1}\right) \geq \frac{1}{4}$ from Lemma 5 . Thus

$\operatorname{mes} E\left\{\theta: \theta_{\nu_{1}+1}<\theta<\theta_{\nu_{1}+2}, \log \left|f\left(R_{k} e^{i \theta}\right)-a_{0}\right|<-\left(C_{0} / 4\right) T\left(R_{k}, f\right)\right\}>2 \alpha$.

Applying Lemma 3 to $f(z), a_{1}$ and the angle $\theta_{\nu_{1}+1}<\arg z<\theta_{\nu_{1}+2}$ which is the neighbor angle of $\theta_{\nu_{1}}<\arg z<\theta_{\nu_{1}+1}$, we get

$$
\log \left|f(z)-a_{1}\right|<-\left(C_{0}^{2} / 4\right) T\left(R_{k}, f\right)
$$

in $\bar{A}\left(10^{-4 Q} R_{k}, 10^{4 Q} R_{k} ; \theta_{\nu_{1}+1}+\alpha, \theta_{\nu_{1}+2}-\alpha\right)$.

By the same deduction, we have

$$
\log \left|f(z)-a_{1}\right|<-\left(C_{0} / 4\right)^{2} T\left(R_{k}, f\right)
$$

on $\left(|z|=R_{k}\right) \cap\left(\theta_{\nu_{1}+2}-\alpha<\arg z<\theta_{\nu_{1}+2}+2 \alpha\right)$ and

$$
\log \left|f(z)-a_{1}\right|<-\left(C_{0} / 4\right)^{2} C_{0} T\left(R_{k}, f\right)
$$


in $\bar{A}\left(10^{-4 Q} R_{k}, 10^{4 Q} R_{k} ; \theta_{\nu_{1}+2}+\alpha, \theta_{\nu_{1}+3}-\alpha\right)$.

Applying this procedure in succession through $\nu_{2}-\nu_{1}(\leq q)$ times, we obtain finally

$$
\begin{aligned}
\log \left|f(z)-a_{1}\right| & <-\left(C_{0} / 4\right)^{\nu_{2}-\nu_{1}} C_{0} T\left(R_{k}, f\right) \\
& \leq-\left(C_{0} / 4\right)^{q} C_{0} T\left(R_{k}, f\right)
\end{aligned}
$$

in $\bar{A}\left(10^{-4 Q} R_{k}, 10^{4 Q} R_{k} ; \theta_{\nu_{2}}+\alpha, \theta_{\nu_{2}+1}-\alpha\right)$. It is clear that the inequality (7.6) contradicts (7.2).

From the above procedure, we know that a point $z_{1}$ in the region

$$
\bar{A}\left(10^{-2 Q} R_{k}, 10^{2 Q} R_{k} ; \theta_{\nu_{1}+h_{1}}-\alpha, \theta_{\nu_{1}+h_{1}}+5 \alpha\right) \quad\left(1 \leq h_{1} \leq \nu_{2}-\nu_{1}\right)
$$

can be found such that

$$
\log \left|f\left(z_{1}\right)-a_{1}\right|>0
$$

and that in the region $\bar{A}\left(10^{-2 Q} R_{k}, 10^{2 Q} R_{k} ; \theta_{\nu_{1}+1}-\alpha, \theta_{\nu_{1}+h_{1}}-\alpha\right) \log \left|f(z)-a_{1}\right| \leq 0$ holds uniformly. Thus we have

$$
\log \left|f(z)-a_{1}\right|<-C_{0}\left(C_{0} / 4\right)^{h_{1}} T\left(R_{k}, f\right)
$$

on the segment $\Gamma_{1}^{\prime}:\left(10^{-4 Q} R_{k} \leq|z| \leq 10^{4 Q} R_{k}\right) \cap\left(\arg z=\theta_{\nu_{1}+h_{1}}-\alpha\right)$.

7.3. Further, there exists a point $z_{1}^{*}$ on the closure of

such that

$$
D_{k 1}^{\prime}: A\left(10^{-4 Q} R_{k}, 10^{4 Q} R_{k} ; \theta_{\nu_{1}+h_{1}}-\alpha, \theta_{\nu_{1}+h_{1}}+11 \alpha\right)
$$

$$
\log \left|f\left(z_{1}^{*}\right)\right|>\left(C_{0} / 4\right)^{q+2} T\left(R_{k}, f\right),
$$

where $q$ is the number of Borel directions of order $\geq \mu$.

In fact, let

$$
\left|f\left(z_{1}^{*}\right)-a_{1}\right|=\max _{z \in \bar{D}_{k 1}^{\prime}}\left|f(z)-a_{1}\right|
$$

and denote by $C \Gamma_{1}^{\prime}$ the complement of $\Gamma_{1}^{\prime}$ with respect to the boundary of $D_{k 1}^{\prime}$. We obtain from (7.7), (7.8), (7.10) and Lemma 6 that

$$
\begin{aligned}
0< & \log \left|f\left(z_{1}\right)-a_{1}\right|<\omega\left(z_{1}, \Gamma_{1}^{\prime}, D_{k 1}^{\prime}\right) \log M\left(\Gamma_{1}^{\prime}, f-a_{1}\right) \\
& +\left\{1-\omega\left(z_{1}, \Gamma_{1}^{\prime}, D_{k 1}^{\prime}\right)\right\} \log M\left(C \Gamma_{1}^{\prime}, f-a_{1}\right) \\
< & \frac{1}{4}\left\{-C_{0}\left(C_{0} / 4\right)^{h_{1}} T\left(R_{k}, f\right)\right\}+\log \left|f\left(z_{1}^{*}\right)-a_{1}\right| .
\end{aligned}
$$

Therefore the inequality (7.9) follows immediately.

If the angle $\theta_{\nu_{3}}<\arg z<\theta_{\nu_{3}+1}$ corresponds to $f^{(-1)}(z)$ and the deficient value $b_{1}$, then we have by Lemma 4 that $\log \left|f^{(-1)}(z)-b_{1}\right|<-C_{0} T\left(R_{k}, f\right)$ and $\log |f(z)|<-\left(C_{0} / 2\right) T\left(R_{k}, f\right)$ in the region $\bar{A}\left(10^{-4 Q} R_{k}, 10^{4 Q} R_{k} ; \theta_{\nu_{3}}+\alpha, \theta_{\nu_{3}+1}-\alpha\right)$. By the same deduction, we obtain a point $z_{2}^{*}$ in

$$
\bar{A}\left(10^{-4 Q} R_{k}, 10^{4 Q} R_{k} ; \theta_{\nu_{2}+h_{2}}-\alpha, \theta_{\nu_{2}+h_{2}}+11 \alpha\right) \quad\left(1 \leq h_{2} \leq \nu_{3}-\nu_{2}\right)
$$

with $\log \left|f\left(z_{2}^{*}\right)\right|>\left(C_{0} / 4\right)^{q+2} T\left(R_{k}, f\right)$.

If the angle $\theta_{\nu_{4}}<\arg z<\theta_{\nu_{4}+1}$ corresponds to $f^{(-1)}(z)$ and the deficient value $b_{2}$, then we also obtain a point $z_{3}^{*}$ in

$$
\bar{A}\left(10^{-4 Q} R_{k}, 10^{4 Q} R_{k} ; \theta_{\nu_{3}+h_{3}}-\alpha, \theta_{\nu_{3}+h_{3}}+11 \alpha\right) \quad\left(1 \leq h_{3} \leq \nu_{4}-\nu_{3}\right)
$$

with $\log \left|f^{(-1)}\left(z_{3}^{*}\right)\right|>\left(C_{0} / 4\right)^{q+2} T\left(R_{k}, f\right)$. 
In general, there is a point $z_{n}^{*}$ in

$$
\bar{A}\left(10^{-4 Q} R_{k}, 10^{4 Q} R_{k}, \theta_{\nu_{n}+h_{n}}-\alpha, \theta_{\nu_{n}+h_{n}}+11 \alpha\right) \quad\left(1 \leq h_{n} \leq \nu_{n+1}-\nu_{n}\right)
$$

satisfying either

$$
\log \left|f\left(z_{n}^{*}\right)\right|>\left(C_{0} / 4\right)^{q+2} T\left(R_{k}, f\right)
$$

or

$$
\log \left|f^{(-1)}\left(z_{n}^{*}\right)\right|>\left(C_{0} / 4\right)^{q+2} T\left(R_{k}, f\right) .
$$

7.4. If $C_{0}$ is given by (5.5), we can find a number $C_{0}^{\prime}$ such that

$$
\frac{1}{4}\left(C_{0} / 4\right)^{q+2} \leq C_{0}^{\prime} \leq \frac{1}{2}\left(C_{0} / 4\right)^{q+2}
$$

and that $f^{\prime}(z)$ has no zero on the level curves $|f(z)|=C_{0}^{\prime} T\left(R_{k}, f\right)$ which are thus analytic.

Consider the set

$$
\Omega:\left\{z: \log |f(z)|>C_{0}^{\prime} T\left(R_{k}, f\right),|z|<10^{4 V} R_{k}\right\},
$$

where $V$ is given by (6.6). Let $\Omega_{k 1}$ be a component of $\Omega$ which contains the point $z_{1}^{*}$. It is clear from Lemma 7, (7.1) and (7.3) that $\Omega_{k 1}$ does not meet $|z|=10^{-4 V} R_{k}$, $\arg z=\theta_{\nu_{1}+1}-\alpha$ and $\arg z=\theta_{\nu_{2}}+\alpha$. But $\bar{\Omega}_{k 1} \cap\left(|z|=10^{4 V} R_{k}\right)$ contains at least one arc by the maximum modulus theorem.

Let $r \varphi_{k 1}(r)$ be the linear measure of $\Omega_{k 1} \cap(|z|=r)$ and let $\Gamma_{k 1}$ be $\bar{\Omega}_{k 1} \cap(|z|=$ $10^{4 V} R_{k}$ ) and $C \Gamma_{k 1}$ be the complement of $\Gamma_{k 1}$ with respect to the boundary of $\Omega_{k 1}$. When $\left|z_{1}^{*}\right| \leq r<10^{4 V} R_{k}$, we have

$$
\begin{aligned}
\log \left|f\left(z_{1}^{*}\right)\right| \leq & \left\{1-\omega\left(z_{1}^{*}, \Gamma_{k 1} ; \Omega_{k 1}\right)\right\} \max _{z \in C \Gamma_{k 1}} \log |f(z)| \\
& +\omega\left(z_{1}^{*}, \Gamma_{k 1} ; \Omega_{k 1}\right) \max _{z \in \Gamma_{k 1}} \log |f(z)| .
\end{aligned}
$$

In view of (7.9), (7.13), (7.14) and an estimation [8, p. 116] of harmonic measure, we obtain

$$
\begin{aligned}
\left(C_{0} / 4\right)^{q+2} T\left(R_{k}, f\right)< & \log \left|f\left(z_{1}^{*}\right)\right|<\frac{1}{2}\left(C_{0} / 4\right)^{q+2} T\left(R_{k}, f\right) \\
& +\log M\left(10^{4 V} R_{k}, f\right) 9 \sqrt{2} \exp \left\{-\pi \int_{2\left|z_{1}^{*}\right|}^{10^{4 V} R_{k} / 2} \frac{d r}{r \varphi_{k 1}(r)}\right\} .
\end{aligned}
$$

Thus

$$
\begin{aligned}
\pi \int_{2 \cdot 10^{4 Q} R_{k}}^{10^{4 V} R_{k} / 2} \frac{d r}{r \varphi_{k 1}(r)}< & \log T\left(2 \cdot 10^{4 V} R_{k}, f\right) \\
& \quad-\log T\left(R_{k}, f\right)+\log \left\{54 \sqrt{2}\left(4 / C_{0}\right)^{q+2}\right\}
\end{aligned}
$$

In the general case, if (7.11) holds, then there is a component $\Omega_{k n}$ of $\Omega$ which contains the point $z_{n}^{*}$ and does not meet $|z|=10^{-4 V} R_{k}, \arg z=\theta_{\nu_{n}+1}-\alpha$ and $\arg z=\theta_{\nu_{n}+1}+\alpha$. Let $r \varphi_{k n}(r)$ be the linear measure of $\Omega_{k n} \cap(|z|=r)$. Then we have the same inequality (7.15) with $\varphi_{k n}(r)\left(n=1,2, \ldots, n_{1}\right)$.

Similarly, there is $C_{0}^{\prime \prime} T\left(R_{k}, f\right)$ in

$$
\left[\frac{1}{4}\left(C_{0} / 4\right)^{q+2} T\left(R_{k}, f\right), \frac{1}{2}\left(C_{0} / 4\right)^{q+2} T\left(R_{k}, f\right)\right]
$$


such that $f(z)$ has no zero on the level curves $\left|f^{(-1)}(z)\right|=C_{0}^{\prime \prime} T\left(R_{k}, f\right)$ which are thus analytic.

Consider the set

$$
\Omega^{\prime}:\left\{z: \log \left|f^{(-1)}(z)\right|>C_{0}^{\prime \prime} T\left(R_{k}, f\right),|z|<10^{4 V} R_{k}\right\} .
$$

If (7.12) holds, let $\Omega_{k n}^{\prime}$ be a component of $\Omega^{\prime}$ which contains the point $z_{n}^{*}$. We can see that $\Omega_{k n}^{\prime}$ does not meet $|z|=10^{-4 V} R_{k}$, arg $z=\theta_{\nu_{n}+1}-\alpha$ and $\arg z=\theta_{\nu_{n+1}}+\alpha$. Let $r \varphi_{k n}^{*}(r)$ be the linear measure of $(|z|=r) \cap \Omega_{k n}^{\prime}$. Then, if $\left|z_{n}^{*}\right| \leq r<10^{4 V} R_{k}$, we have

$$
\begin{aligned}
& \left(C_{0} / 4\right)^{q+2} T\left(R_{k}, f^{(-1)}\right)<\log \left|f^{(-1)}\left(z_{n}^{*}\right)\right|<\frac{1}{2}\left(C_{0} / 4\right)^{q+2} T\left(R_{k}, f^{(-1)}\right) \\
& \quad+\log M\left(10^{4 V} R_{k}, f^{(-1)}\right) \cdot 9 \sqrt{2} \exp \left\{-\pi \int_{2\left|z_{n}^{*}\right|}^{10^{4 V} R_{k} / 2} \frac{d r}{r \varphi_{k n}^{*}(r)}\right\} .
\end{aligned}
$$

Since

$$
\begin{aligned}
\log M\left(10^{4 V} R_{k}, f^{(-1)}\right) & \leq \log M\left(10^{4 V} R_{k}, f\right)+O\left(\log 10^{4 V} R_{k}\right) \\
& <4 T\left(2 \cdot 10^{4 V} R_{k}, f\right)
\end{aligned}
$$

and

$$
\begin{aligned}
T\left(R_{k}, f\right) & \leq T\left(R_{k}, f^{(-1)}\right)+m\left(R_{k}, \frac{f}{f^{(-1)}}\right) \\
& =T\left(R_{k}, f^{(-1)}\right)+O\left(\log R_{k}\right)<\frac{4}{3} T\left(R_{k}, f^{(-1)}\right),
\end{aligned}
$$

this yields that

$$
\begin{aligned}
\pi \int_{2 \cdot 10^{4 Q} R_{k}}^{10^{4 V} R_{k} / 2} \frac{d r}{r \varphi_{k n}^{*}(r)}< & \log T\left(2 \cdot 10^{4 V} R_{k}, f\right) \\
& -\log T\left(R_{k}, f\right)+\log \left\{\begin{array}{c}
\left.144 \sqrt{2}\left(\frac{4}{C_{0}}\right)^{q+2}\right\} . \\
n=1,2, \ldots, n_{2} .
\end{array}\right.
\end{aligned}
$$

Therefore we obtain finally $n_{1}$ components $\Omega_{k n}\left(n=1,2, \ldots, n_{1}\right)$ of $\Omega$ and $n_{2}$ components $\Omega_{k n}^{\prime}\left(n=1,2, \ldots, n_{2}\right)$ of $\Omega^{\prime}$ and the corresponding inequalities (7.15) with $\varphi_{k n}(r)$ and (7.16). It is very important to note that $n_{1}+n_{2}=p_{0}+p_{-1}$ and that any one of $\Omega_{k n}\left(n=1,2, \ldots, n_{1}\right)$ and $\Omega_{k n}^{\prime}\left(n=1,2, \ldots, n_{2}\right)$ is distant from all others. Thus

$$
\sum_{n=1}^{n_{1}} \varphi_{k n}(r)+\sum_{n=1}^{n_{2}} \varphi_{k n}^{*}(r) \leq 2 \pi
$$

so that

$$
\begin{aligned}
\left(p_{0}+p_{-1}\right)^{2} & =\left\{\sum_{n=1}^{n_{1}}\left(\varphi_{k n}(r)\right)^{1 / 2} \frac{1}{\left(\varphi_{k n}(r)\right)^{1 / 2}}+\sum_{n=1}^{n_{2}}\left(\varphi_{k n}^{*}(r)\right)^{1 / 2} \frac{1}{\left(\varphi_{k n}^{*}(r)\right)^{1 / 2}}\right\}^{2} \\
& \leq\left\{\sum_{n=1}^{n_{1}} \varphi_{k n}(r)+\sum_{n=1}^{n_{2}} \varphi_{k n}^{*}(r)\right\}\left\{\sum_{n=1}^{n_{1}} \frac{1}{\varphi_{k n}(r)}+\sum_{n=1}^{n_{2}} \frac{1}{\varphi_{k n}^{*}(r)}\right\} \\
& \leq 2 \pi\left\{\sum_{n=1}^{n_{1}} \frac{1}{\varphi_{k n}(r)}+\sum_{n=1}^{n_{2}} \frac{1}{\varphi_{k n}^{*}(r)}\right\}
\end{aligned}
$$


Consequently, we have

$$
\begin{aligned}
& \frac{\left(p_{0}+p_{-1}\right)^{2}}{2} \int_{2 \cdot 10^{4 Q} R_{k}}^{10^{4 V} R_{k} / 2} \frac{d r}{r} \\
& \quad \leq \sum_{n=1}^{n_{1}} \pi \int_{2 \cdot 10^{4 Q} R_{k}}^{10^{4 V} R_{k} / 2} \frac{d r}{r \varphi_{k n}(r)}+\sum_{n=1}^{n_{2}} \pi \int_{2 \cdot 10^{4 Q} R_{k}}^{10^{4 V} R_{k} / 2} \frac{d r}{r \varphi_{k n}^{*}(r)} \\
& \leq\left(p_{0}+p_{-1}\right)\left\{\log T\left(2 \cdot 10^{4 V} R_{k}, f\right)-\log T\left(R_{k}, f\right)\right\} \\
& \quad+\left(p_{0}+p_{-1}\right) \log \left\{144 \sqrt{2}\left(4 / C_{0}\right)^{q+2}\right\} .
\end{aligned}
$$

But

$$
t_{k} \leq R_{k} \leq 2 t_{k}, \quad T\left(4 \cdot 10^{4 V} t_{k}, f\right)<\left\{\left(8 \cdot 4 \cdot 10^{4 V}\right)^{\mu}+o(1)\right\} T\left(t_{k}, f\right)
$$

and $T\left(R_{k}, f\right) \geq T\left(t_{k}, f\right)$ by Lemma 2 and Lemma 1 with $\beta_{1}=8, \beta_{2}=4 \cdot 10^{4 V}$ and therefore we obtain

$$
\left(p_{0}+p_{-1}\right) \log \frac{10^{4 V}}{4 \cdot 10^{4 Q}} \leq 2 \log \left(33 \cdot 10^{4 V}\right)^{\mu}+2 \log \left\{144 \sqrt{2}\left(\frac{4}{C_{0}}\right)^{q+2}\right\} .
$$

Letting $V \rightarrow+\infty$, we have $p_{0}+p_{-1} \leq 2 \mu$.

If $p_{0}$ or $p_{-1}$ equals $+\infty$, then we can choose $p_{0}^{\prime}$ deficient values of $f(z)$ and $p_{-1}^{\prime}$ deficient values of $f^{(-1)}(z)$ such that $p_{0}^{\prime}+p_{-1}^{\prime}=[2 \mu]+1$. The same procedure, however, leads to $p_{0}^{\prime}+p_{-1}^{\prime} \leq 2 \mu$. This contradiction shows that both $p_{0}$ and $p_{-1}$ must be finite. When $p_{0}=0$ or $p_{-1}=0$, the above deduction is also effective. Thus the proof of the theorem is complete.

ACKNOWLEDGMENTS. This work was partially supported by the National Science Foundation of China. I would like to thank the referee for the useful opinions.

\section{REFERENCES}

1. N. U. Arakelyan, Entire functions of finite order with an infinite set of deficient values, Dokl. Akad. Nauk SSSR 170 (1966), 999-1002.

2. D. Drasin, Proof of a conjecture of $F$. Nevanlinna concerning functions which have deficiency sum two, Acta Math. 158 (1987), 1-94.

3. _ Quasi-conformal modifications of functions having deficiency sum two, Ann. of Math. 114 (1981), 493-518.

4. A. Edrei and W. H. J. Fuchs, Valeurs déficientes et valeurs asymptotiques des fonctions méromorphes, Comment. Math. Helv. 33 (1959), 258-295.

5. W. K. Hayman, Meromorphic functions, Oxford, 1964.

6. R. Nevanlinna, Analytic functions, Springer-Verlag, 1970.

7. A. Pfluger, Zur Defektrelation ganzer Funktionen endlicher ordnung, Comment. Math. Helv. 19 (1946), $91-104$.

8. M. Tsuji, Potential theory in modern function theory, Maruzen, Tokyo, 1959.

9. G. Valiron, Directions de Borel des fonctions méromorphes, Mém. Sci. Math., fasc. 89, Paris, 1938.

10. A. Weitsman, Meromorphic functions with maximal deficiency sum and a conjecture of $F$. Nevanlinna, Acta Math. 123 (1969), 115-139.

11. __ A growth property of the Nevanlinna characteristic, Proc. Amer. Math. Soc. 26 (1970), 6570.

12. Yang Lo, (Growth and angular distribution of entire functions (preprint).

13. Yang Lo and Zhang Kuan-heo, Sur la distribution des directions de Borel des fonctions méromorphes, Sci. Sinica 16 (1973), 465-482. 